J. Lake Sci.(湖泊科学), 2019, 31(1): 147-158

DOI 10. 18307/2019. 0114

(c) 2019 by Journal of Lake Sciences

\title{
阳宗海砷污染背景下浮游植物的时空分布特征及其驱动因子解析”
}

\author{
白宁静 ${ }^{1}$, 陈 丽 $^{1 * *}$, 蒋伊能 ${ }^{1}$, 张 涛 $^{1}$, 刘 术 $^{1}$, 刘晓䂀 ${ }^{1}$, 李天 丽 $^{2}$, 赵帅营 ${ }^{1}$, 陈光杰 ${ }^{1}$ \\ ( 1 : 云南师范大学旅游与地理科学学院, 高原地理过程与环境变化省重点实验室, 昆明 650500$)$ \\ (2: 中国科学院水生生物研究所,武汉 430072)
}

\begin{abstract}
摘 要: 人类活动导致的重金属污染是湖泊水体面临的主要环境压力之一. 云南高原湖泊阳宗海于 2008 年暴发了砷污 染事件且水体砷浓度目前仍然超过生活饮用水卫生标准, 严重影响了水安全和生态系统健康. 本研究于 2015 年 4 月2016 年 2 月每两月对阳宗海南、中、北部湖区进行采样调查及分析, 共鉴定出浮游植物 7 门 44 属 68 种, 绿藻门种数最多. 蓝藻门占绝对优势, 其中伪鱼腥藻 (Pseudoanabaena sp.) 、浮丝藻 (Planktothrix sp.) 、束丝藻 (Aphanizomenon sp.) 为全年的 优势种, 这与已有调查显示的阳宗海砷污染后浮游植物群落中蓝藻占优的基本特征一致. 方差分析结果表明浮游植物生 物量在时间尺度上呈现显著的变化特征, 最大值出现在 8 月 ( $14.06 \mathrm{mg} / \mathrm{L})$, 最小值出现在 12 月 $(1.23 \mathrm{mg} / \mathrm{L})$, 而空间差异 不显著. Pearson 相关分析显示, 浮游植物总生物量与水温、 $\mathrm{pH}$ 呈显著正相关, 而与砷、透明度、锰、钠、钾和总磷浓度呈显 著负相关. 冗余分析结果表明,水温、砷、钙、锰、钾共同解释了阳宗海浮游植物群落结构变化的 $57.18 \%$. 方差分解的结果 进一步表明, 水温、钙离子和砷三者作用共同解释了浮游植物群落结构变化的 $32.05 \%$, 其中水温和钻离子分别独立解释 了群落变化的 $12.45 \%$ 和 $8.28 \%$, 水体砷浓度仅独立解释了 $2.33 \%$, 但与水温共同作用解释了 $9.46 \%$. 因此, 我们推测水温 的季节性波动导致了湖泊水体热力分层的明显变化, 其中水体混合作用的增强可能会促进底泥释放过程并增加表层水 体的砷浓度, 进而影响了浮游植物群落的季节性变化. 研究结果有利于评价重金属污染对湖泊的长期生态效应, 并为砷 污染湖泊的环境修复提供重要的科学依据.
\end{abstract}

关键词: 阳宗海; 砷污染; 浮游植物; 群落结构; 环境因子

\section{Spatio-temporal characteristics of phytoplankton distribution and the identification of driving factors in the arsenic-contaminated Yangzong Lake}

BAI Ningjing ${ }^{1},{\text { CHEN } \mathrm{Li}^{1 * *} \text {, JIANG Yineng }}^{1}$, ZHANG Tao ${ }^{1}$, LIU Shu ${ }^{1}$, LIU Xiaoxi ${ }^{1}$, LI Tianli ${ }^{2}$, ZHAO Shuaiying ${ }^{1} \&$ CHEN Guangjie ${ }^{1}$

(1: Provincial Key Laboratory of Plateau Geographical Processes and Environmental Change, School of Tourism and Geography, Yunnan Normal University, Kunming 650500, P.R.China)

(2: Institute of Hydrobiology, Chinese Academy of Sciences, Wuhan 430072, P.R.China)

\footnotetext{
Abstract: Heavy metal pollution induced by human activities is widely regarded as a major environmental stressor for many lakes. Yangzong Lake, one of the nine largest plateau lakes in Yunnan Province, has experienced severe arsenic contamination since 2008. Monitoring data further showed that lake-water arsenic concentration consistently exceeded the standards for drinking water quality, posing severe risk for water safety and ecosystem health. A spatial survey and analysis of surface water quality and phytoplankton in Yangzong Lake was conducted bimonthly from April 2015 to February 2016, covering the south, central and north parts of the lake basin, respectively. A total of 68 algal taxa were identified, with the largest number of taxa belonging to Chlorophyta. However, Cyanophyta was absolutely dominant throughout the study period, and consisted of Pseudoanabaena, Planktothrix and Aphanizomenon. These results were consistent with previous surveys of phytoplankton from this lake, confirming the cyanobacteriadominated community structure after the occurrence of arsenic pollution. The analysis of variance demonstrated that the phytoplank-

* 国家自然科学基金项目 $(41461096,41771239,31460131) 、 2017$ 年中西部高等学校青年骨干教师国内访问学者项 目和云南省应用基础研究计划项目面上项目 (2018FB077) 联合资助. 2018-04-16 收稿; 2018-05-16 收修改稿. 白宁静(1993 ),女,硕士研究生;E-mail: m18180103093@ qq.com.

** 通信作者;E-mail: chenli5311@163.com.
} 
ton biomass exhibited a significant seasonal fluctuation, with a maximum value found in August (14.06 mg/L) and a minimum biomass occurring in December $(1.23 \mathrm{mg} / \mathrm{L})$, while no significant spatial variation was observed. The Pearson correlation analysis showed that phytoplankton total biomass was significantly and positively correlated with water temperature and $\mathrm{pH}$, but negatively correlated with arsenic concentration, water transparency, manganese concentration, sodium concentration, potassium concentration and total phosphorus concentration. Redundancy analysis displayed that water temperature, arsenic concentration, calcium concentration, manganese concentration and potassium concentration altogether accounted for $57.18 \%$ of the community variation in Yangzong Lake. The variation partitioning further showed that the water temperature, calcium concentration and arsenic concentration accounted for $32.05 \%$ of the spatio-temporal variation of phytoplankton community in Yangzong Lake, while the water temperature, calcium concentration and arsenic concentration independently accounted for $12.45 \%, 8.28 \%$ and $2.33 \%$ of the total variance, respectively. The coupling of arsenic concentration and water temperature explained $9.46 \%$ of the community variation. Therefore, we infer that seasonal fluctuation of water temperature may have caused the change in lake stratification and the increased water mixing under lower temperature may promote the sediment release of arsenic, resulting in higher lake-water arsenic concentration and stronger community turnover of phytoplankton. This study will help to evaluate the long term ecological effect of heavy metals on lakes and provide scientific evidence for restoration of arsenic polluted plateau lakes.

Keywords: Yangzong Lake; arsenic contamination; phytoplankton; community structure; environmental factor

重金属污染是云南地区湖泊和水库面临的主要环境压力之一 ${ }^{[1-2]}$. 由于重金属在自然环境中较难降解, 并且具有普遍的生物毒性、生物累积性和生物放大作用,因此水体的重金属污染会对水资源安全与生态系 统健康产生持续的危害. 砷是一种毒性较高且致癌、致畸的类金属污染物, 在水体中的停留时间长达 50 年 ${ }^{[3]}$. 工业排放等人类活动将大量的砷释放到环境中, 增大了砷在水体中的分布范围和浓度, 同时威胁着水 体水质和人类健康 ${ }^{[4-5]}$. 阳宗海是云南九大高原湖泊之一,于 2008 年暴发了严重的砷污染事件,湖水中砷浓 度在 12 月高达 $176.9 \mu \mathrm{g} / \mathrm{L}^{[6]}$. 经过多年的综合治理和生态修复, 水体中的砷浓度呈现下降且相对平稳的趋 势, 但是仍然超过 $10 \mu \mathrm{g} / \mathrm{L}$ 的生活饮用水卫生标准 ${ }^{[5-7]}$. 因此, 阳宗海水体中较高的砷浓度对湖泊生态系统 健康和社会经济持续发展构成长期的潜在危害.

浮游植物是淡水生态系统的重要组分和湖泊食物网的关键环节, 在物质循环和能量流动中起着重要的 作用 ${ }^{[8]}$. 浮游植物的种类组成和生物量在不同的水生生态系统中均存在着较大的差异 ${ }^{[9]}$, 其群落结构和功 能特征与水体光照、营养盐、水深等环境因子关系密切,环境条件的变化会影响浮游植物的结构组成、优势 种及生物量等群落特征 ${ }^{[10]}$. 已有研究表明, 砷污染会造成浮游植物优势种发生转变并引起群落结构的变 化 ${ }^{[11-12]}$, 同时重金属污染物可经浮游植物等初级生产者通过食物链改变生态系统的结构和功能, 从而导致 湖泊生物多样性的降低和生态系统稳定性的下降 ${ }^{[13]}$. 如对阳宗海硅藻群落沉积物记录的调查发现, 随着砷 污染的出现, 部分藻类物种数量大量减少, 而耐砷污染的硅藻迅速转变为优势种, 同时浮游动物生物量出现 10 倍以上的下降, 湖泊生态系统出现了灾难性的转变 ${ }^{[14]}$. 因此, 阳宗海重金属污染可能导致浮游植物群落 结构发生改变进而对湖泊食物链结构和生态系统稳定性产生重要影响.

阳宗海浮游植物的现代调查始于 1950s, 已有结果反映了藻类优势种属和群落结构存在显著的季节变 化和长期演替特征. 1957 年的调查显示, 阳宗海浮游植物主要包括菱形圆盘硅藻 (Cyclotella rhomboideo-elliptica) (硅藻门)、蓝隐藻 (Chroomonas sp.) ( 隐藻门)、飞燕角甲藻 (Ceratium hirundinella) ( 甲藻门)、勃氏黄被 藻 (Botryococcus) (黄藻门) 和伸臂角星鼓藻 (Staurastrum paradoxom) (绿藻门) ${ }^{[15]}$. 在 2005- 2006 年期间, 浮 游植物优势种全年以微囊藻属 (Microcystis sp.) (蓝藻门) 为主 ${ }^{[16]} .2007$ 和 2008 年的调查发现, 藻类优势种 出现了明显的季节波动, 如在 3 月份以卵囊藻属 (Oocystis sp.) (绿藻门) 为主, 而 7 月和 11 月分别以脆杆藻 属 (Fragilaria sp.) (硅藻门)、并联藻属 ( Quadrigula sp.) (绿藻门) 和微囊藻属为主 ${ }^{[17]}$. 随着砷污染的出现与 持续, 阳宗海浮游植物优势种均为蓝藻门, 微囊藻、拟鱼腥藻 (Anabaenopsis sp.) 、尖头藻 (Raphidiopsis sp.) 和 束丝藻 (Aphanizomenon sp.) 交替出现成为 2009-2010 年的优势种 ${ }^{[18]}$, 而席藻 (Phormidium sp.) 则在 2011 年 全年占优 ${ }^{[17]} .2013$ 年的调查发现, 阳宗海浮游植物的优势种群为蓝藻门的拟鱼腥藻和泽丝藻 ( Limnothrix

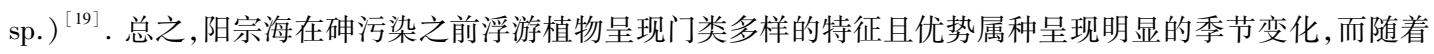
砷污染的出现与持续, 浮游植物以蓝藻门占绝对优势, 而其他门类的数量大量减少. 目前对阳宗海砷污染的 
环境治理已有多年但水体污染问题仍然持续, 急需系统识别阳宗海浮游植物群落的时空变化特征, 并甄别 水体砷污染持续的背景下驱动浮游植物群落构建的关键因子.

本研究以云南典型砷污染湖泊阳宗海为对象, 于 2015 年 4 月- 2016 年 2 月对浮游植物及主要环境因 子进行系统调查, 通过分析阳宗海浮游植物群落结构、生物量和优势种的时空变化并对其变化的驱动因子 进行解析, 评价砷在胁迫环境中的生态作用, 以期为有效开展污染湖泊的环境治理和生态修复提供科学 依据.

\section{1 材料与方法}

\section{1 研究区域概况及采样点设置}

阳宗海 ( $24^{\circ} 01^{\prime} \sim 24^{\circ} 58^{\prime} \mathrm{N}, 102^{\circ} 59^{\prime} \sim 103^{\circ} 02^{\prime} \mathrm{E}$ ) 地跨宜良、澄江、呈贡三县, 两端略宽, 中间稍窄; 平均水 位 $1770.6 \mathrm{~m}$ 时, 南北平均长 $12 \mathrm{~km}$, 东西平均宽 $2.7 \mathrm{~km}$, 湖岸线长 $32.3 \mathrm{~km}$, 湖区面积 $31.7 \mathrm{~km}^{2}$, 最大水深 30 $\mathrm{m}$, 平均水深 $19.5 \mathrm{~m}$, 蓄水量 $6.04 \times 10^{8} \mathrm{~m}^{3}$, 流域面积 $192 \mathrm{~km}^{2[20]}$. 阳宗海是典型的高原构造型亚热带石灰岩淡 水深水湖泊, 属珠江流域南盘江水系, 该流域属北亚热带季风气候, 受西南季风影响明显, 多年平均气温 $14.5^{\circ} \mathrm{C}$, 平均降水量 $963.5 \mathrm{~mm}, 6-10$ 月降水量占年降水量的 $87 \%$. 主要人湖河流有南部的阳宗河、七里河以 及东西两侧的暂时性溪流, 东北部的汤池河为该湖唯一的出水口 ${ }^{[20-21]}$. 阳宗海周边分布着多个泉眼,地下水 补给是该湖重要的补给方式 ${ }^{[22]} .2006$ 年之前, 阳宗海水体砷浓度不足 $2 \mu \mathrm{g} / \mathrm{L}^{[23]}, 2007$ 年 9 月略有升高,但 未超过 $6 \mu \mathrm{g} / \mathrm{L}$ 的水平 ${ }^{[24]} .2008$ 年 10-12 月阳宗海湖水中平均砷浓度为 $167 \mu \mathrm{g} / \mathrm{L}^{[25]}, 12$ 月高达 $176.9 \mu \mathrm{g} /$ $\mathrm{L}, 2009$ 年平均砷浓度为 $155.9 \mu \mathrm{g} / \mathrm{L}^{[6]}$. 同时对阳宗海沉积物的研究显示, 沉积物中砷浓度背景值(如 1950 年以前) 约为 $34.4 \mu \mathrm{g} / \mathrm{g}$, 在 1990s 初开始逐渐增加, 并且在 2007- 2008 年附近达到最高值 $479 \mu \mathrm{g} / \mathrm{g}$, 与 1950 年前的背景时期相比阳宗海沉积物的砷浓度升高了将近 14 倍, 与湖泊监测记录反映的湖泊污染历史一 致 ${ }^{[14]}$. 随着湖泊治理的开展, 湖水砷浓度呈现下降的趋势, 2010 年 4 月阳宗海平均砷浓度为 $82.7 \mu \mathrm{g} / \mathrm{L}^{[5]}$, 2013 年水体的砷浓度为 $54.3 \mu \mathrm{g} / \mathrm{L}$, 是生活饮用水卫生标准的 5 倍以上 ${ }^{[7]}$.

根据阳宗海湖泊形态设置南、中、北 3 个采样点, 分别为 $\mathrm{T} 1\left(24^{\circ} 52^{\prime} 11^{\prime \prime} \mathrm{N}, 102^{\circ} 59^{\prime} 29^{\prime \prime} \mathrm{E}\right) 、 \mathrm{~T} 3\left(24^{\circ} 54^{\prime} 24^{\prime \prime} \mathrm{N}\right.$, $\left.103^{\circ} 00^{\prime} 20^{\prime \prime} \mathrm{E}\right)$ 和 T5 $\left(24^{\circ} 57^{\prime} 31^{\prime \prime} \mathrm{N}, 103^{\circ} 00^{\prime} 59^{\prime \prime} \mathrm{E}\right)$, 如图 1 所示. 本次调查采样时间为 2015 年 4 月- 2016 年 2 月,每 2 月采集一次,共计 6 次采样.

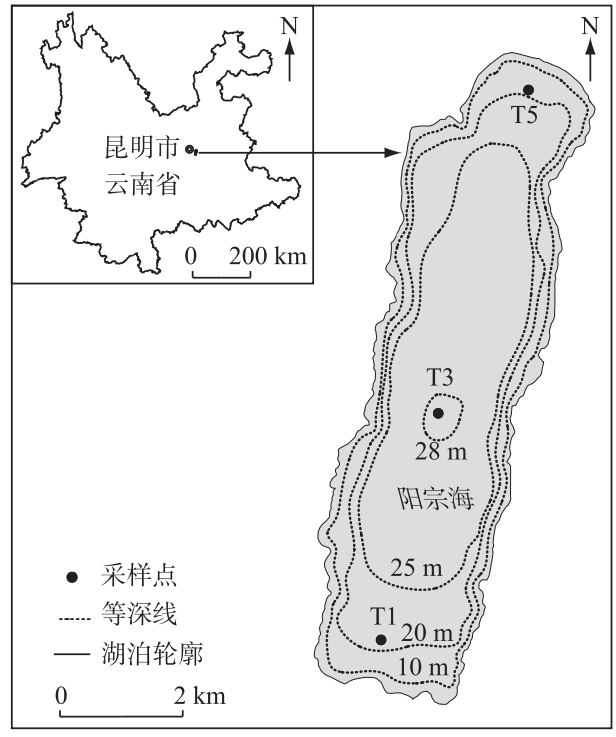

图 1 阳宗海采样点分布

Fig.1 Distribution of sampling sites in Yangzong Lake 


\section{2 样品采集与处理}

浮游植物定性样品用孔径为 $20 \mu \mathrm{m}$ 的浮游生物网采集, 将采集的样品装人 $60 \mathrm{ml}$ 样品瓶中, 加人鲁哥和 甲醛摇匀, 带回实验室用 Leica 光学显微镜在 400 倍下进行鉴定. 将样品经酸处理后制成固定玻片在 1000 倍油镜下进行硅藻的鉴定. 定量样品用 $5 \mathrm{~L}$ 的有机玻璃采水器采集表层以下 $0.5 \mathrm{~m}$ 处的水样, 取 $1 \mathrm{~L}$ 水样现 场加人鲁哥和甲醛摇匀之后带回实验室静置 $48 \mathrm{~h}$, 用虹吸管吸取上清液, 浓缩至 $30 \mathrm{ml}$, 完全摇匀后, 取 0.1 $\mathrm{ml}$ 藻液到浮游生物计数框中, 在光学显微镜下计数, 采用形态相似的几何体积的方法计算出浮游植物的体 积, 从而转换为生物量 ${ }^{[26-27]}$.

采用 YSI 6600 现场测定水温、pH、溶解氧和电导率, 用塞氏盘测定水体透明度. 总磷和总氮浓度的测定 方法参照《水与废水监测分析方法》 (第四版) 进行 ${ }^{[28]}$. 水体中的砷、镁、钠、钻、钾、铅、铁和锰的浓度根据国 家环境保护标准( GB7475－1987、GB7477－1987、GB7485－1987、GB11904－1989、GB11911－1989、HJ/T3422007、HJ/T343-2007) 进行测定.

\section{3 数据分析}

阳宗海浮游植物优势度 $(Y)$ 的计算公式为:

$$
Y=\left(n_{i} / N\right) f_{i}
$$

式中, $n_{i}$ 为第 $i$ 种物种的个体数, $N$ 为所有物种的总个体数, $f_{i}$ 为第 $i$ 种物种出现的频率. 物种优势度 $Y \geqslant$ 0.02 定为优势种.

本研究采用 Pearson 相关分析分析阳宗海浮游植物的生物量与环境因子的相互关系, 利用相似性分析 (analysis of similarities, ANOSIM) 检验浮游植物群落结构在时空上的差异性, 利用方差分析 (analysis of variance, ANOVA) 分析浮游植物生物量及环境因子的时空差异, 利用午余分析 (redundancy analysis, RDA) 研究 浮游植物群落结构与环境因子的相互关系: 首先对群落结构百分比数据进行去趋势对应分析 (detrended correspondence analysis, DCA), 结果显示梯度长度最大为 1.34 , 因此选择线性分析中的圥余分析方法; 其次对各 物种的生物量百分比数据进行平方根转换, 提高稀有种在群落结构中的百分比; 对各环境因子进行正态分 布检验, 并且进行不同的转换使其趋于正态分布; 最后将转换后的环境数据进行独立的 RDA 分析, 将显著 的环境因子 $(P<0.05)$ 与浮游植物生物量百分比进行 RDA 排序. 将 $P<0.05$ 的环境因子进行方差膨胀因子 的检验 (Variance inflation factor, VIF), 剔除膨胀系数大于 20 的环境因子之后对剩余的环境因子进行简约模 型分析, 最后得到最为显著地解释浮游植物变化的环境因子 ${ }^{[29]}$, 并将显著的环境因子和群落数据进行方差 分解 (Variation Partitioning).

本文的数据分析在 Excel、Origin8.0 及 R (3.2.2) 软件中的 vegan、multcomp 和 psych 软件包中进行.

\section{2 结果}

\section{1 浮游植物群落结构及优势种}

本次研究共鉴定出浮游植物 7 门 44 属 68 种, 其中绿藻门种类最多, 有 15 属 23 种, 占总种类数的 $33.82 \%$; 硅藻门 11 属 22 种, 占 $32.35 \%$; 蓝藻门 12 属 13 种, 占 $19.12 \%$; 隐藻门 2 属 4 种, 占 $5.88 \%$; 甲藻门 2 属 4 种, 占 $5.88 \%$; 金藻门和裸藻门 1 属 1 种,各占 $1.47 \%$.

采样期间蓝藻门生物量基本占绝对优势, 其次为甲藻门, 甲藻门生物量百分比在冬春季节相对高; 硅藻 门生物量百分比在 2015 年 10 月开始增加, 到 12 月达到最大; 其他门类的藻类所占百分比全年均较低 (图 2 ). 蓝藻门的伪鱼腥藻 (Pseudoanabaena sp.) 、浮丝藻 (Planktothrix sp.) 和束丝藻为全年的优势种, 棒胶藻 (Rhabdogloea sp.) 在 2015 年 4-12 月为主要优势种, 中华小尖头藻 (Merismopedia sinica) 在 6、8 和 10 月为优 势种, 弯形小尖头藻 (Raphidiopsis curvata) 在 $6 、 8$ 和 2 月为优势种. 绿藻门、硅藻门和隐藻门的个别物种在某 些月份成为优势种, 如微小四角藻 (Tetraedron minimum) 、小环藻 (Cyclotella spp.)、脆杆藻 (Fragilaria spp.) 及 蓝隐藻 (Chroomonas sp.) 在 2 月成为优势种; 极小曲壳藻 (Achnanthes minutissima) 为 10 和 12 月的优势种. 2016 年 2 月的优势种种类最多, 4 月种类最少 (表 1). AONSIM 结果显示阳宗海浮游植物群落结构在时间上 差异显著 $(r=0.55, P<0.01)$; 空间上, 南、中、北 3 个湖区差异不显著 $(r=-0.12, P=0.97)$. 


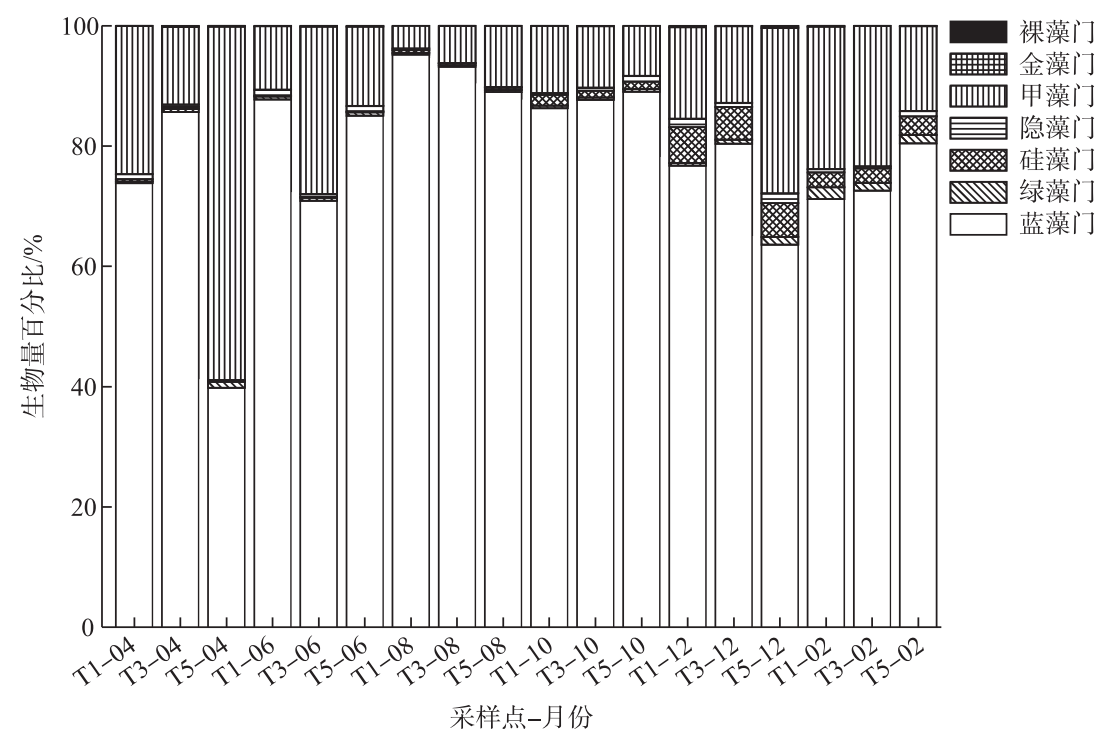

图 2 阳宗海浮游植物群落结构的时空变化

Fig.2 Spatio-temporal variation of phytoplankton community structure by biomass in Yangzong Lake

表 1 阳宗海各月份浮游植物优势种及优势度对比

Tab.1 Summary of dominant algal taxa and their dominance in Yangzong Lake

\begin{tabular}{|c|c|c|c|c|c|c|c|}
\hline \multirow{2}{*}{ 门类 } & \multirow{2}{*}{ 优势种 } & \multicolumn{6}{|c|}{ 优势度 } \\
\hline & & 4 月 & 6 月 & 8 月 & 10 月 & 12 月 & 2 月 \\
\hline 蓝藻 & 伪鱼腥藻 (Pseudoanabaena sp.) & 0.68 & 0.36 & 0.19 & 0.30 & 0.62 & 0.17 \\
\hline 蓝藻 & 浮丝藻 ( Planktothrix sp.) & 0.13 & 0.09 & 0.06 & 0.11 & 0.08 & 0.37 \\
\hline 蓝藻 & 束丝藻 (Aphanizomenon sp.) & 0.06 & 0.16 & 0.32 & 0.15 & 0.08 & 0.15 \\
\hline 蓝藻 & 棒胶藻( Rhabdogloea sp.) & 0.05 & 0.31 & 0.31 & 0.35 & 0.07 & - \\
\hline 蓝藻 & 中华小尖头藻 (Merismopedia sinica) & - & 0.03 & 0.06 & 0.03 & - & - \\
\hline 蓝藻 & 弯形小尖头藻 (Raphidiopsis curvata) & - & 0.04 & 0.04 & - & - & 0.07 \\
\hline 绿藻 & 微小四角藻 ( Tetraedron minimum) & - & - & - & - & - & 0.02 \\
\hline 硅藻 & 小环藻 (Cyclotella spp.) & - & - & - & - & - & 0.07 \\
\hline 硅藻 & 脆杆藻 ( Fragilaria spp.) & - & - & - & - & - & 0.04 \\
\hline 硅藻 & 极小曲壳藻( Achnanthes minutissima) & - & - & - & 0.02 & 0.08 & - \\
\hline 隐藻 & 蓝隐藻 ( Chroomonas sp.) & - & - & - & - & - & 0.03 \\
\hline
\end{tabular}

“_”表示优势度 $<0.02$.

\section{2 浮游植物生物量}

阳宗海南部和中部湖区浮游植物总生物量在时间上呈现显著的季节性变化趋势, 即从 2015 年 4-8 月 逐渐升高, 8 月达到最高, 之后 8-12 月出现显著下降, 2016 年 2 月又相对上升. 北部湖区浮游植物生物量 在 4 月最高, 6 和 8 月略有下降, 而在其他月份与南部和中部湖区的变化趋势一致. 总生物量最大值出现在 南部湖区的 8 月 (14.06 mg/L), 最小值出现在南部湖区的 12 月 ( $1.23 \mathrm{mg} / \mathrm{L})$ ( 图 3). 浮游植物、蓝藻门和硅 藻门生物量在时间上存在显著差异 ( ANOVA, $n=18, P<0.001$ ), 而其他门类的藻类生物量在时间上的差异不 显著; 空间上, 阳宗海南、中、北部湖区浮游植物和各门类的生物量差异均不显著 $(\mathrm{ANOVA}, n=18, P>0.05)$.

\section{3 浮游植物及其驱动因子}

阳宗海水体主要环境指标显示 (表 2), 除铁的浓度外, 其他环境因子在时间尺度上均存在着显著的差异 


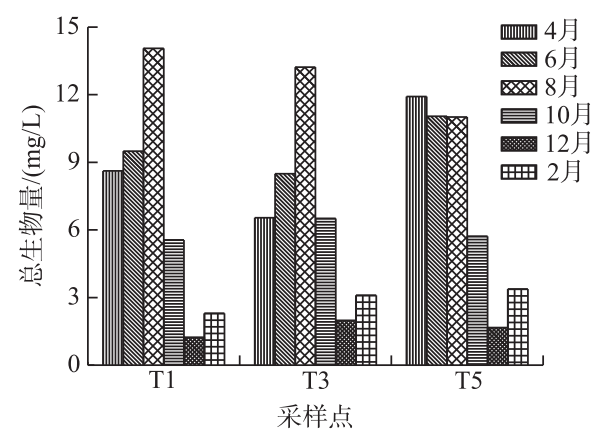

图 3 阳宗海浮游植物生物量的时空分布

Fig.3 Spatio-temporal distribution of phytoplankton biomass in Yangzong Lake

$(n=18, P<0.05)$; 对 3 个湖区各环境指标的差异进行 ANOVA 检验, 结果显示各环境指标的空间变化差异不 显著 $(n=18, P>0.05)$.

表 2 阳宗海水体主要环境指标特征

Tab.2 Summary of environmental factors of Yangzong Lake

\begin{tabular}{lccccc}
\hline 指标 & 平均值土标准差 & 数值范围 & 指标 & 平均值标准差 & 数值范围 \\
\hline 水温 $/{ }^{\circ} \mathrm{C}$ & $19.16 \pm 4.52$ & $11.52 \sim 25.09$ & 砷 $/(\mu \mathrm{g} / \mathrm{L})$ & $48.19 \pm 22.27$ & $7.00 \sim 78.20$ \\
$\mathrm{pH}$ & $8.46 \pm 0.55$ & $7.35 \sim 9.13$ & 钲 $/(\mathrm{mg} / \mathrm{L})$ & $18.37 \pm 15.81$ & $7.43 \sim 52.90$ \\
溶解氧 $/(\mathrm{mg} / \mathrm{L})$ & $7.66 \pm 1.54$ & $4.87 \sim 9.35$ & 镁 $/(\mathrm{mg} / \mathrm{L})$ & $22.92 \pm 5.98$ & $8.52 \sim 32.20$ \\
透明度 $/ \mathrm{m}$ & $1.69 \pm 0.88$ & $0.78 \sim 4.90$ & 钠 $/(\mathrm{mg} / \mathrm{L})$ & $4.99 \pm 1.41$ & $2.24 \sim 6.84$ \\
总氮 $/(\mu \mathrm{g} / \mathrm{L})$ & $989.32 \pm 297.82$ & $670.00 \sim 1830.66$ & 钾 $/(\mathrm{mg} / \mathrm{L})$ & $3.25 \pm 1.14$ & $1.37 \sim 4.46$ \\
总磷 $/(\mu \mathrm{g} / \mathrm{L})$ & $41.55 \pm 8.19$ & $30.00 \sim 53.40$ & 铁 $/(\mu \mathrm{g} / \mathrm{L})$ & $5.94 \pm 7.57$ & $0.50 \sim 32.20$ \\
电导率 $/(\mathrm{ms} / \mathrm{cm})$ & $0.36 \pm 0.04$ & $0.28 \sim 0.40$ & 锰 $/(\mu \mathrm{g} / \mathrm{L})$ & $2.79 \pm 2.22$ & $0.25 \sim 6.80$ \\
铅 $/(\mu \mathrm{g} / \mathrm{L})$ & $1.21 \pm 0.98$ & $0.05 \sim 3.82$ & & & \\
\hline
\end{tabular}

在阳宗海浮游植物总生物量与环境因子的相关关系上, 浮游植物总生物量与水温和 $\mathrm{pH}$ 呈显著正相关 $(P<0.001)$; 与砷、锰、透明度 $(P<0.001)$ 及钠、钾和总磷 $(P<0.05)$ 呈显著负相关. 由于蓝藻和硅藻生物量对 浮游植物群落结构的贡献较大且在时间上具有显著的差异, 故将两者分别与环境因子进行相关性分析. 结 果显示, 蓝藻生物量与水温、 $\mathrm{pH}(P<0.001)$ 及铅 $(P<0.05)$ 呈显著正相关; 与砷、锰、透明度 $(P<0.001)$ 及钠、 总磷 $(P<0.05)$ 呈显著负相关. 硅藻生物量与总磷 $(P<0.001)$ 以及锰、电导和总氮 $(P<0.05)$ 呈显著正相关, 而 与钙 $(P<0.01)$ 及水温、 $\mathrm{pH}$ 、溶解氧 $(P<0.05)$ 呈显著负相关 $($ 附录 I ).

进一步采用 RDA 综合判别阳宗海藻类群落结构与环境因子之间的关系. 首先对各环境因子进行独立 的 RDA 分析, 结果表明水温、钲、锰、砷、 $\mathrm{pH}$ 和钾是影响浮游植物群落结构的 6 个显著环境因子 $(P<0.05)$. 浮游植物的变化也直接影响了水体 $\mathrm{pH}$ 水平, 故选取其他 5 个显著的环境因子开展 RDA 的进一步分析, 5 个 环境因子共同解释了浮游植物群落结构变化的 57.18\%. 选取物种得分大于 0.6 的物种与显著的环境因子生 成 RDA 排序图 (图 4), 图中主轴 1 和主轴 2 共解释了浮游植物群落结构变化的 $41.42 \%$, 其中主轴 1 解释了 $26.77 \%$, 主轴 2 解释了 $14.65 \%$. 结果表明阳宗海浮游植物群落结构在时间上的变化幅度明显大于空间上. 从季节性分布来看, 2015 年 4-12 月的浮游植物群落样点主要沿主轴 2 变化, 同时 2016 年 2 月的浮游植物 分布沿主轴 1 变化, 与其他 5 个月的分布特征明显不同. 2016 年 2 月的样点分布在主轴 1 的负半轴, 个别绿 藻和硅藻的出现与水体中较高的砷、钾、锰浓度相关且受到水温总体偏低的影响. 2015 年 $6 、 8 、 10$ 月样点主 要位于主轴 1 和主轴 2 的正半轴, 蓝藻门的束丝藻、尖头藻和棒胶藻的生物量百分比较高, 这与较高的水温 和较低的砷、钾和锰浓度有关. 而 2015 年 4 和 12 月的样点都位于主轴 2 的负半轴, 表明其群落结构主要受 水体中较高的钙、锰和砷浓度影响. 


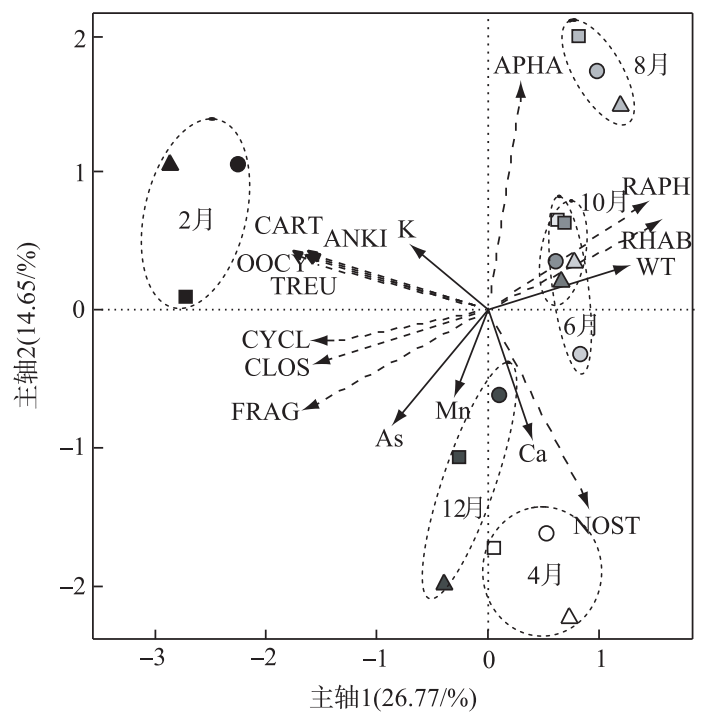

图 4 阳宗海浮游植物群落结构与 5 个显著环境因子的 RDA 排序图

（符号方框、圆圈和三角形分别表示采样点 $\mathrm{T} 1$ 、 $\mathrm{T} 3$ 和 $\mathrm{T} 5$; 物种名: APHA 束丝藻、RAPH 尖头藻、

RHAB 棒胶藻、NOST 念珠藻、CYCL 小环藻、FRAG 脆杆藻、CLOS 新月藻、TREU 四棘藻、 OOCY 卵囊藻、CART 四鞭藻、ANKI 纤维藻; 环境因子: WT 水温、Ca 钲、Mn 锰、As 砷、K 钾)

Fig.4 RDA triplot showing phytoplankton community structure and five significant environmental factors in Yangzong Lake

经 VIF 检验和简约模型分析后, 从上述 5 个显著的 环境因子中进一步选出水温、钙和砷 3 个环境指标, 与 浮游植物群落结构数据结合进行方差分解分析. 结果显 示, 水温、钙和砷共同解释了浮游植物群落结构变化的 $32.05 \%$, 水温、钙和砷分别单独解释了浮游植物群落结 构变化的 $12.45 \% 、 8.28 \%$ 和 $2.33 \%$, 水温和砷共同解释 了浮游植物变化的 $9.46 \%$, 水温和钙共同解释了浮游植 物变化的 $0.89 \%$, 钙和砷共同解释了浮游植物变化的 $0.58 \%$ (图 5).

\section{3 讨论}

\section{1 浮游植物群落结构的变化特征}

本研究共鉴定出浮游植物 7 门 44 属 68 种, 其中绿 藻门种类最多, 硅藻门和蓝藻门次之, 其他门类种类较 少. 蓝藻门为主要优势种, 这与阳宗海砷污染以来浮游 植物优势种的变化特征一致 ${ }^{[17-19]}$. 本次调查结果显示, 大部分点位的蓝藻生物量占浮游植物总生物量的 70\% 以上 (图 2), 反映了蓝藻对砷胁迫可能具有较强的耐受

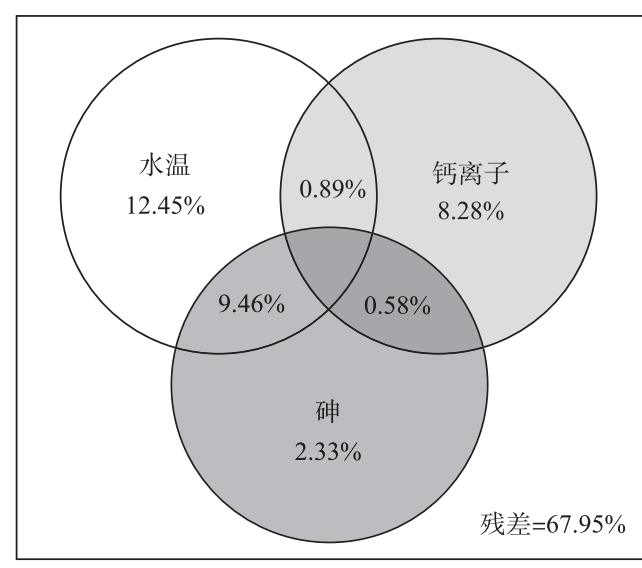

图 5 阳宗海浮游植物群落结构与 环境因子的方差分析

Fig.5 Variation partitioning results showing the explanatory powers of water temperature, calcium and arsenic in accounting for phytoplankton community changes in Yangzong Lake 性 $^{[30]}$. 近几十年来, 阳宗海周边大力发展旅游业, 并且

新建了大量的工矿企业, 同时流域土地利用强度的增加都导致了湖区承载的污染物总量逐渐增加 ${ }^{[7]}$, 阳宗 海面临着富营养化的长期影响, 这也可能导致蓝藻生物量在全年占优. 此外, 蓝藻与其他藻类相比具有较强 
的竞争优势, 蓝藻种类的大量繁殖往往会分泌化感物质抑制其他藻类的生长, 从而降低其他藻类的生物 量 $^{[31]}$. 甲藻门生物量所占比例仅次于蓝藻门, 尤其在冬春季具有较高的水平; 阳宗海出现的甲藻为飞燕角 甲藻和多甲藻, 由于其个体体型大且在全年均有出现, 故生物量百分比在全年占有较大比例. 已有研究表明 甲藻适宜生长的温度范围是 $10 \sim 20^{\circ} \mathrm{C}^{[32]}$, 阳宗海冬春季节水体温度在 $10 \sim 20^{\circ} \mathrm{C}$ 的范围内, 有利于甲藻的生 长; 在冬春季节适宜的温度条件下, 多数甲藻能通过自身调节, 进行垂直迁移获取光照和营养盐, 从而具有 更大的竞争优势 ${ }^{[33-34]}$; 此外, 阳宗海冬春季节甲藻相对较多可能与冬春季水体混合, 把底层部分大个体的甲 藻带到表层, 使表层甲藻生物量增加有关. 硅藻的生长对于水体温度的要求相对于蓝藻低 ${ }^{[35-36]}$, 同时高原湖 泊较强的光照强度和水体营养盐浓度的上升可能有利于个体较小的中心纲硅藻生长 ${ }^{[37]}$, 并且冬春季节水体 混合强度的增加也会促进硅藻含量增加 ${ }^{[38]}$, 这些都可能导致阳宗海硅藻生物量在 12 和 2 月相对较高.

\section{2 阳宗海浮游植物驱动因子解析}

$\mathrm{RDA}$ 和方差分解的分析结果表明, 水温是影响阳宗海浮游植物群落结构季节变化的显著因子. 水温是 影响浮游植物个体生长的关键环境因子之一, 影响着浮游植物组成及时空分布 ${ }^{[39]}$. 水温可以通过对浮游植 物光合作用与呼吸代谢速率的控制而影响藻类含量 ${ }^{[40]}$, 同时大多数藻类随着水温的升高而快速生长和繁 殖, 而水温的下降将导致藻类生长变缓或者停滞 ${ }^{[41]}$. 阳宗海浮游植物总生物量与水温呈显著正相关 (附录 I ). 阳宗海 $6 、 8$ 和 10 月浮游植物生物量较高, 平均水温分别为 $23.8 、 24.3$ 和 $20.4^{\circ} \mathrm{C}$. 而 $4 、 12$ 月和 2016 年 2 月表层平均水温均低于 $20^{\circ} \mathrm{C}$, 浮游植物的生长相对受到抑制, 生物量较低. Paerl 等的研究进一步表明, 当水 体温度大于 $25^{\circ} \mathrm{C}$ 时, 蓝藻的生长相对于硅藻和绿藻更具优势 ${ }^{[35-36]}$; 而李媂等的研究显示当平均水温大于 $20^{\circ} \mathrm{C}$ 时有利于蓝藻生长 ${ }^{[42]}$. 阳宗海浮游植物群落结构显示, 束丝藻、尖头藻和棒胶藻等喜高温的蓝藻含量在 $6 、 8$ 和 10 月相比其他季节增加. 而阳宗海冬季水温相对其他季节低, 特别是 2 月平均水温仅为 $11.5^{\circ} \mathrm{C}$, 同时 群落结构出现了较大的变化, 反映了蓝藻的生长相对受到抑制而硅藻和绿藻的生物量百分比有所增加.

国内外研究表明, 砷对浮游植物的细胞生长和光合活性均具有重要影响 ${ }^{[43-44]}$. 不同种类的藻对不同形 态及不同浓度砷的敏感性和耐受程度不同 ${ }^{[14,30]}$, 因此水体砷浓度的变化对浮游植物群落组成的构建可能产 生差异性的影响 ${ }^{[4,11]}$. 研究显示长期砷胁迫能够改变浮游植物的物种组成, 导致对砷敏感的种类数量减少 甚至消失, 浮游植物优势种向能够获得竞争优势的抗砷种类转变 ${ }^{[1-113]}$. 与海洋藻类相比, 淡水藻类对砷的响 应更加敏感, 如在营养盐限制的淡水环境中自然本底的砷浓度可以影响微藻群落的构建 ${ }^{[12]}$. 研究显示不同 营养条件下砷对不同株系铜绿微囊藻 (蓝藻) 的影响不同, 如无磷或者磷限制 (约 $31 \mu \mathrm{g} / \mathrm{L}$ ) 条件下, 铜绿微 囊藻 FACHB 905 对砷的耐受阈值是 $7.5 \mu \mathrm{g} / \mathrm{L}^{[45]} ; 75 \mu \mathrm{g} / \mathrm{L}$ 的砷在无磷、磷限制、磷富集 ( $\left.310 \mu \mathrm{g} / \mathrm{L}\right)$ 以及磷过 量 $(5425 \mu \mathrm{g} / \mathrm{L})$ 的条件下都能对铜绿微囊藻 FACHB 905 产生抑制作用, 且抑制作用随着磷浓度的增加而减 小 ${ }^{[46]}$; 而铜绿微囊藻 PCC7806 和 TY-1 对高达 $7500 \mu \mathrm{g} / \mathrm{L}$ 的砷耐受, 且耐受性与胞外磷含量无关 ${ }^{[47-48]}$. 调查 期间阳宗海水体砷浓度范围为 $7.0 \sim 78.2 \mu \mathrm{g} / \mathrm{L}$ (平均砷浓度为 $48.2 \mu \mathrm{g} / \mathrm{L}$ ), 磷浓度的范围为 $30.0 \sim 53.4 \mu \mathrm{g} /$ $\mathrm{L}$, 平均值为 $41.6 \mu \mathrm{g} / \mathrm{L}$ (表 2). 相关分析结果表明, 阳宗海浮游植物总生物量和蓝藻生物量与砷浓度均呈显 著负相关 (附录 I ). 在上述的水体磷浓度范围内, 阳宗海砷污染对藻类特别是蓝藻的生长可能产生抑制作 用, 与龚艳等 ${ }^{[45]}$ 和 Guo 等 ${ }^{[46]}$ 的结果一致. 同时, 在水体营养水平较高的背景下 (表 2), 阳宗海砷污染水体中 蓝藻优势的维持机制以及其受砷与营养盐的驱动作用还有待于室内控制实验等研究的进一步开展.

本研究中水体砷浓度的季节变化与水温呈显著负相关 (附录 I ), 方差分解的结果显示砷浓度仅独立解 释了浮游植物群落结构变化的 $2.33 \%$, 而砷和水温共同解释了群落结构变化的 $9.46 \%$. 阳宗海为亚热带深水 湖泊, 其水温具有较强的季节性变化, 热力分层也呈现出季节性特征 ${ }^{[49]}$. 阳宗海夏季水温较高, 湖泊产生强 烈的热力分层阻碍上下水体的交换, 湖上层和湖下层的溶解氧、 $\mathrm{pH}$ 、电导率、氧化还原电位等产生显著的变 化 ${ }^{[21,50]}$, 并且可能使水体底部内源释放的砷无法带到湖上层, 出现了湖泊表层水体相对较低的砷浓度, 而在 冬季, 湖泊水体温度梯度小、热力混合强度增加, 导致了水体物理与化学环境特征在垂直分布上趋于同质 化, 同时内源释放的砷等污染物进人水体表层导致水体砷浓度升高 ${ }^{[49-50]}$, 故阳宗海表层砷浓度存在较强的 季节性变化特征 (表 2). 因此, 我们推测在长期砷污染湖泊中, 水温的季节性波动导致了湖泊热力分层的变 化并改变了表层水体中的砷浓度, 从而可能对浮游植物生物量和群落结构的季节变化产生影响.

云贵高原具有广泛分布的喀斯特地貌, 大量分布的石灰岩、白云岩和泥灰岩中钲、钾、镁和钠等阳离子 
含量较高 ${ }^{[51]}$. 钲作为藻类生长的一种必需的营养元素, 是藻类生长发育的重要调节因子, 能够维持细胞壁、 细胞膜及膜结合蛋白的稳定性, 还可作为第二信使调节植物的多种代谢过程. 一定浓度的外源钙对重金属 胁迫下藻类的生长发育、生理代谢、抗氧化系统、超微结构等均具有明显的改善作用, 从而增强藻类对重金 属毒害环境的适应性 ${ }^{[52]}$. 部分研究表明, 水体中铝离子浓度的降低将降低藻类的生长速率、最大光电子传 递速率及水体的叶绿素浓度, 但过高浓度的钙离子也会抑制藻类的生长 ${ }^{[53-54]}$. 另外, 研究显示不同门类的浮 游植物对于水体中不同钙离子浓度的响应不同 ${ }^{[55-56]}$, 故钻离子对于浮游植物群落结构的构建在一定程度上 起到了调节作用. 本次调查中, 钙离子的浓度变化范围较大 $(7.4 \sim 52.9 \mathrm{mg} / \mathrm{L})$, 同时 RDA 结果表明钙离子浓 度与阳宗海 4 和 12 月浮游植物群落结构显著相关, 方差分解结果也显示钙离子能够解释浮游植物群落结构 变化的 $9.75 \%$. 因此在喀斯特地质背景下, 钲离子浓度等水体化学特征是云贵高原地区湖泊中浮游植物群 落构建的重要影响因子.

\section{4 结论}

1) 采样期间阳宗海浮游植物以蓝藻门占绝对优势, 其中伪鱼腥藻、浮丝藻、束丝藻为全年优势种, 且优 势度较大.

2) 阳宗海浮游植物生物量整体上在春夏季高, 秋冬季低. 除铁离子以外各环境指标和浮游植物生物量 均在时间上存在显著差异, 而空间差异不显著. 水温、 $\mathrm{pH}$ 、砷、透明度、锰、钠、钾和总磷浓度是影响浮游植物 总生物量变化的主要环境因子.

3 ) 水温、钙和砷是影响阳宗海浮游植物群落结构季节变化的重要环境因子. 我们推测砷主要通过水温 的变化对浮游植物群落产生作用, 在较低水温的情况下, 湖泊水体在垂直方向上的季节性混合促进了砷的 内源释放, 表层水体砷浓度的升高可能对浮游植物群落的构建产生直接影响. 本研究结果可以为胁迫环境 中砷的生态作用评价以及砷污染湖泊的综合治理提供一定的数据支持和理论基础.

致谢: 感谢高原地理过程与环境变化省重点实验室各位老师和同学在野外采样和室内分析中给予的帮助.

\section{5 附录}

附录见电子版 (DOI: 10.18307/2019.0114).

\section{6 参考文献}

[ 1 ] Lui ZT, Li CH, Zhang GY. Application of principal component analysis to the distributions of heavy metals in the water of lakes and reservoirs in Yunnan Province. Research of Environmental Sciences, 2010, 23(4) : 459-466. [刘总堂, 李春 海, 章钢娅. 运用主成分分析法研究云南湖库水体中重金属分布. 环境科学研究, 2010, 23(4) : 459-466.]

[ 2 ] Xing W, Bai G, Wu H et al. Effect of submerged macrophytes on metal and metalloid concentrations in sediments and water of the Yunnan Plateau lakes in China. Journal of Soils \& Sediments, 2017, 17(10) : 1-10.

[ 3 ] Klein DH. Fluxes, residence times, and sources of some elements to Lake Michigan. Water Air \& Soil Pollution, 1975,4 (1) : 3-8.

[ 4 ] Knauer K, Behra R, Hemond H. Toxicity of inorganic and methylated arsenic to algal communities from lakes along an arsenic contamination gradient. Aquatic Toxicology, 1999, 46(3/4) : 221-230.

[ 5 ] Zhang YX, Xiang XP, Zhang Y et al. Distribution and Sources in Yangzonghai Lake, China. Environmental Science, 2012, 33(11) : 3768-3777. [张玉胥, 向小平, 张英等. 云南阳宗海砷的分布与来源. 环境科学, 2012, 33(11): 3768-3777.]

[ 6 ] Wang ZH, He B, Pan XJ et al. Levels, trends and risk assessment of arsenic pollution in Yangzonghai Lake, Yunnan Province, China. Science China Chemistry, 2010, 53(8) : 1809-1817.

[ 7 ] Tao JS, Chen GJ, Chen XL et al. Long-term pattern of diatom community responses to water pollution and hydrological regulation in Yangzong Lake. Geographical Research, 2016, 35(10): 1899-1911. [陶建霜, 陈光杰, 陈小林等. 阳宗海硅 藻群落对水体污染和水文调控的长期响应模式. 地理研究, 2016, 35(10): 1899-1911.]

[ 8 ] Reynolds CS ed. The ecology of freshwater phytoplankton. Cambridge: Cambridge University Press, 2006.

[ 9 ] Lu N, Yin HB, Deng JC et al. Spring community structure of phytoplankton from Lake Chaohu and its relationship to envi- 
ronmental Factors. J Lake Sci, 2010, 22(6) : 950-956. DOI: 10.18307/2010.0620. [ 路娜, 尹洪斌, 邓建材等. 巢湖流 域春季浮游植物群落结构特征及其与环境因子的关系. 湖泊科学, 2010, 22(6)：950-956.]

[10] Suikkanen S, Laamanen M, Huttunen M. Long-term changes in summer phytoplankton communities of the open northern Baltic Sea. Estuarine Coastal \& Shelf Science, 2007, 71(3/4) : 580-592.

[11] Sanders J, Cibik. Adaptive behavior of euryhaline phytoplankton communities to arsenic stress. Marine Ecology Progress, 1985, 22 : 199-205.

[12] Wängberg S, Heyman U, Blanck H. Long-term and short-term arsenate toxicity to freshwater phytoplankton. Canadian Journal of Fisheries \& Aquatic Sciences, 1991, 48(2) : 173-182.

[13] Sanders JG. Direct and indirect effects of arsenic on the survival and fecundity of estuarine zooplankton. Canadian Journal of Fisheries \& Aquatic Sciences, 1986, 43(3) : 694-699.

[14] Chen GJ, Shi HB, Tao JS et al. Industrial arsenic contamination causes catastrophic changes in freshwater ecosystems. Scientific Reports, 2015, 5: 17419.

[15] Li SH, Yu MJ, Li GZ et al. Limnological survey of the lakes of Yunnan Plateau. Oceanologia et Limnologia sinica, 1963, 5(2) : 87-114. [ 黎尚豪, 俞敏娟, 李光正等. 云南高原湖泊调查. 海洋与湖沼, 1963, 5(2): 87-114.]

[16] Li YP, Yan X. Changes of phytoplankton biomass of Yangzonghai Lake from 2005 to 2007. Environmental Science Survey, 2015, (s1)：8-10. [李娅萍, 颜翔. 2005-2007 年阳宗海浮游植物生物量变化特征分析. 环境科学导刊, 2015, (s1):8-10.]

[17] Xu YM. Arsenic concentration and phytoplankton change in Lake Yangzong. Environmental Science Survey, 2013, 32(5) : 62-64. [徐永梅. 阳宗海砷浓度与浮游植物的变化分析. 环境科学导刊, 2013, 32(5): 62-64.]

[18] Li CY, Yang ZL. Phytoplankton community and nutritional status evaluation in Yangzonghai Lake. Pearl River, 2013, 3 (6) : 20-23. [ 李春永, 杨中兰. 阳宗海浮游植物群落与营养状态评价. 人民珠江, 2013, 3(6): 20-23.]

[19] Xie YH, Li CY, Yang ZL. Research on plankton community structure of Yangzonghai Lake. Water Resources Protection, 2015, 31(4) : 47-51. [谢永红, 李春永, 杨中兰. 阳宗海浮游生物群落结构研究. 水资源保护, 2015, 31 (4) : 47-51.]

[20］Wang SM, Dou HS eds. Records of lakes in China. Beijing: Science Press, 1998. [王苏民, 窦鸿身. 中国湖泊志. 北 京: 科学出版社, 1998.]

[21] Yuan LN, Yang CL, Li XM et al. Effect of daily thermal stratification on dissolved oxygen, pH, total phosphorus concentration, phytoplankton and algae density of a deep plateau lake: A case study of Lake Yangzonghai, Yunnan Province. J Lake Sci, 2014, 12(1): 161-168. DOI: 10.18307/2014.0120. [袁琳娜, 杨常亮, 李晓铭等. 高原深水湖泊水温日成 层对溶解氧、酸碱度、总磷浓度和藻类密度的影响 : 以云南阳总海为例. 湖泊科学, 2014, 12(1) : 161-168.]

[22] Zhu XH, Lyu SS, Zhang PP et al. Heavy metal contamination in the lacustrine sediment of a plateau lake: influences of groundwater and anthropogenic pollution. Environmental Earth Sciences, 2016, 75(2): 1-14.

[23] Li RF, Li XM, Xu Q et al. Identification of arsenic sources and pollution control in Yangzonghai Lake. Environmental Science Survey, 2015, (5) : 27-31. [李发荣, 李晓铭, 徐琼等. 阳宗海湖泊砷污染来源解析与防治. 环境科学导刊, $2015,(5): 27-31$.

[24] Yin HW. Arsenic Pollution in Yangzong Lake has received extensive attention.South Reviews, 2009, 25(4): 46-49. [尹 鸿伟. 阳宗海砷污染迷雾不散. 南风窗, 2009, 25(4)：46-49.]

[25] Qi JY, Xu ZC, Li XP et al. Study on source and speciation distribution characteristics of arsenic in Yangzonghai Lake waters. Journal of Anhui Agricultural Science, 2010, 38(20): 10789-10792. [齐剑英, 许振成, 李祥平等. 阳宗海水体中 砷的形态分布特征及来源研究. 安徽农业科学, 2010, 38(20) : 10789-10792.]

[26] Ma J, Wang J. Phytoplankton collection, counting and quantitative methods. Journal of Hydroecology, 1982, (4) : 58-63. [马䩀, 王建. 浮游植物的采集、计数与定量方法. 水生态学杂志, 1982, (4) : 58-63.]

[27] Hillebrand H, Dürselen C, Kirschtel D et al. Biovolume calculation for pelagic and benthic microalgae. Journal of Phycolo$g y, 1999, \mathbf{3 5}(2)$ : 403-424.

[28] Editorial board of "water and wastewater monitoring and analysis method”, Ministry of Environmental Protection of the People's Republic of China ed. Minitoring and analysis methods of water and wastewater: fourth edition. Beijing: China Envirinmental Science Press, 2002: 243-285. [ 国家环境保护局《水与废水监测分析方法》编委会. 水和废水监测分 析方法: 第四版. 北京: 中国环境科学出版社, 2002: 243-285.] 
[29] Jiang YN, Zhang T, Chen L et al. Identification of phytoplankton community changes and environmental factors during the winter-spring transition in Fuxian Lake. Transactions of Oceanology and Limnology, 2016, (6) : 87-98. [蒋伊能, 张涛, 陈丽等.抚仙湖冬-春季浮游植物群落结构变化及其影响因子识别. 海洋湖沼通报, 2016, (6) : 87-98.]

[30] Ferrari SG, Silva PG, González DM et al. Arsenic tolerance of cyanobacterial strains with potential use in biotechnology. Revista Argentina de Microbiologia, 2013, 45(3): 174-179.

[31] Yang B, Dong JD, WJ et al. Allelopathy in phytoplankton. Acta Ecologica Sinica, 2007, 27(4): 1619-1626. [杨斌, 董 俊德, 吴军等. 浮游植物的化感作用. 生态学报, 2007, 27(4): 1619-1626.]

[32] Yao XJ, Liu DF, Yang ZJ et al. Preliminary studies on the mechanism of winter dinoflagellate bloom in Xiangxi Bay of the Three Gorges Reservoir. Research of Environmental Sciences, 2012, 25(6) : 645-651. [姚绪姣, 刘德富, 杨正健等. 三峡 水库香溪河库湾冬季甲藻水华生消机理初探. 环境科学研究, 2012, 25(6) : 645-651.]

[33] Xu Y, Cai Q, Wang L et al. Diel vertical migration of Peridiniopsis niei, Liu et al, a new species of dinoflagellates in an eutrophic bay of Three-Gorge Reservoir, China. Aquatic Ecology, 2010, 44(2) : 387-395.

[34] Ross ON, Sharples J. Swimming for survival: A role of phytoplankton motility in a stratified turbulent environment. Journal of Marine Systems, 2008, 70(3/4) : 248-262.

[35] Paerl HW, Huisman J. Blooms like it hot. Science, 2008, 320(5872) : 57-58.

[36] Johnk K, Huisman J, Sharples J et al. Summer heatwaves promote blooms of harmful cyanobacteria. Global Change Biolo$g y, 2008, \mathbf{1 4}(3): 495-512$.

[37] Rolland A, Bertrand F, Maumy M et al. Assessing phytoplankton structure and spatio-temporal dynamics in a freshwater ecosystem using a powerful multiway statistical analysis. Water Research, 2009, 43(13) : 3155.

[38 ] Yang D, Gao Z, Sun P et al. Mechanism of nutrient silicon and water temperature influences on phytoplankton growth. Marine Science Bulletin, 2006, 8(2): 49-59.

[39] Lopes MRM, Bicudo CEDM, Ferragut MC. Short term spatial and temporal variation of phytoplankton in a shallow tropical oligotrophic reservoir, southeast Brazil.Hydrobiologia, 2005, 542(1) : 235-247.

[40] Jin JP, Yu X, Bao YX et al. Study on the relationship between the cyanobacteria density and the previous environmental factors in the east part of the Yangcheng Lake. Urban Meteorology Forum-Urban and Environmental Meteorology, 2014: 324-331. [ 金建平, 于金金, 包云轩等. 阳澄湖东湖蓝藻密度与前期环境因子的关系. 城市气象论坛一城市与环境气 象, 2014: 324-331. ]

[41] Tan X, Kong FX, Yu Y et al. Effects of enhanced temperature on algae recruitment and phytoplankton community succession. China Environment Science, 2009, 29(6) : 578-582. [谭啸, 孔繁翔, 于洋等. 升温过程对藻类复苏和群落演替 的影响. 中国环境科学, 2009, 29(6): 578-582.]

[42] Li D, Li XW, Niu ZC et al. Structure of phytoplankton community and relationship between phytoplankton community and water quality in Taihu Lake. Ecology and Environmental Sciences, 2014, 23(11)：1814-1820. [李媂, 李旭文, 牛至春 等. 太湖浮游植物群落结构及其与水质指标间的关系. 生态环境学报, 2014, 23(11) : 1814-1820.]

[43] Sorsa KK. Culture studies on arsenic (As III, As V, MMAA) interaction with three freshwater algae (Microcystis aeruginosa, Actinastrum hantzschii and Asterionella formosa). Madison: Univ of Wisconsin Madison Wi, 1983.

[44] Wang S, Zhang D, Pan X. Effects of arsenic on growth and photosystem II (PSII) activity ofMicrocystis aeruginosa. Ecotoxicology \& Environmental Safety, 2012, 84(7) : 104.

[45] Gong Y, Wu XQ, Xiao BD et al. Response of Microcystis aeruginosa under different phosphate regimes. Acta Hydrobiologica Sinica, 2009, 33(5) : 890-895. [ 供艳, 吴幸强, 肖邦定等. 铜绿微囊藻在不同供磷水平下对砷胁迫的响应. 水生 生物学报, 2009, 33(5): 890-895.]

[46] Guo P, Gong Y, Wang C et al. Arsenic speciation and effect of arsenate inhibition in a Microcystis aeruginosa culture medium under different phosphate regimes. Environmental Toxicology \& Chemistry, 2011, 30( 8) : 1754-1759.

[47] Gong Y, Chou HN, Tu CD et al. Effects of arsenate on the growth and microcystin production of Microcystis aeruginosa, isolated from Taiwan as influenced by extracellular phosphate. Journal of Applied Phycology, 2009, 21(2) : 225-231.

[48] Gong Y, Song L, Wu X et al. Effects of arsenate on microcystin content and leakage of Microcystis Strain PCC7806 under various phosphate regimes. Environmental Toxicology, 2009, 24(1) : 87-94.

[49] Li ZY. Distribution, morphology and structure of arsenic in Yangzong Lake and its stability in sediments [Dissertation]. Kunming: Yunnan University, 2014. [李智圆. 阳宗海湖泊砷的分布、形态结构及其在沉积物中的稳定性 [ 学位论 
文]. 昆明: 云南大学, 2014.]

[50] Yang CL. Migration and transformation of arsenic in sediments of Yangzong Lake under accelerated sedimentation conditions [Dissertation]. Kunming: Yunnan University, 2014. [ 杨常亮. 人为加速沉积条件下阳宗海沉积物中砷的迁 移转化规律 [学位论文]. 昆明: 云南大学, 2014. ]

[51] Chen XL. Research of lake diatom community and biodiversity distribution feature in Yunnan [Dissertation]. Kunming: Yunnan Normal University, 2015. [陈小林. 云南高原湖泊硅藻群落与生物多样性的分布特征研究 [ 学位论文]. 昆 明: 云南师范大学, 2015.]

[52] Min HL, Cai SJ, Xu QS et al. Effects of exogenous calcium on resistance of Hydrilla verticillata ( L.f.) Royle to cadmium stress. Acta Ecological Sinica, 2012, 32(1) : 256-264. [闵海丽, 蔡三娟, 徐勤松等. 外源钲对黑藻抗镉胁迫能力的 影响. 生态学报, 2012, 32(1): 256-264.

[53] Shi J, Wu Z, Song L. Physiological and molecular responses to calcium supplementation in Microcystis aeruginosa (Cyanobacteria). New Zealand Journal of Marine \& Freshwater Research, 2013, 47 (1) : 51-61.

[54] El-Zahraa F, Zaki T. Effect of boron and calcium on growth and nitrogen fixation of the blue-green alga Calothrix parietina. Folia Microbiologica, 1999, 44(2) : 201-204.

[55] Ding L, Zhi CY.Environmental effects on diatom and its monitor of environment. Journal of Guizhou Normal University: Natural Sciences, 2006, 24(3) : 13-16. [丁蕾, 支崇远. 环境对硅藻的影响及硅藻对环境的监测. 贵州师范大学学 报: 自然版, 2006, 24(3): 13-16.]

[57] Tao ZY, Chen WJ, Yu ZJ et al. Correlation analysis of phytoplankton community structure and environmental factors in Taibo Lake. Journal of Anhui Agricultural Sciences, 2017, 45(13): 63-67. [陶志英, 陈文静, 余智杰等. 太泊湖浮游 植物群落结构特征及其与环境因子相关性分析. 安徽农业科学, 2017, 45(13)：63-67.] 


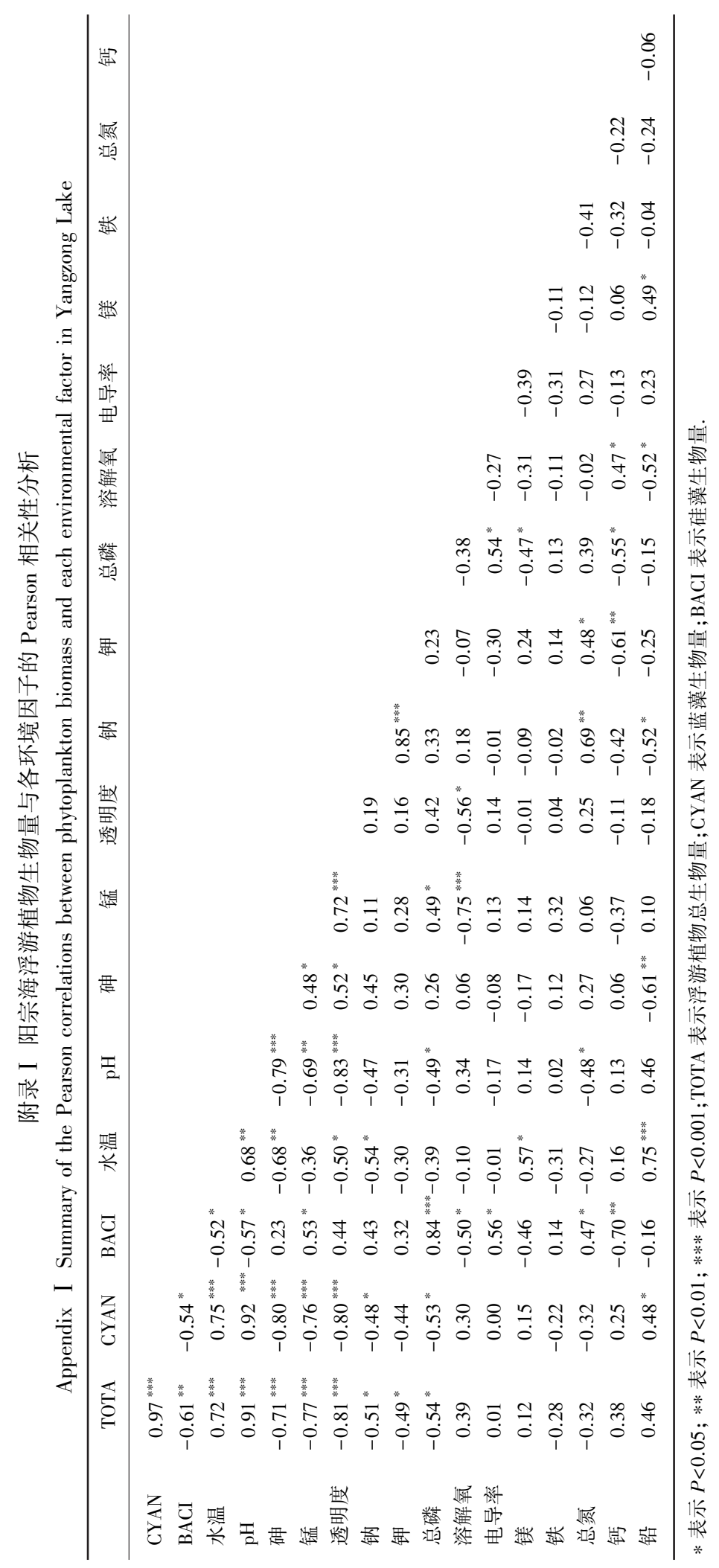

